\title{
Thermo-mechanical Noise Measurement of Sealed Nanobeams on a Silicon Photonics-MEMS Platform
}

\author{
Khannan Rajendran ${ }^{1 *}$, Awanish Pandey ${ }^{1}$, Pierre Edinger ${ }^{2}$, Gaehun $\mathrm{Jo}^{2}$, Alain Yuji Takabayashi ${ }^{3}$, Umar Khan ${ }^{1}$, \\ Peter Verheyen ${ }^{4}$, Niels Quack ${ }^{3}$, Frank Niklaus ${ }^{2}$, Wim Bogaerts ${ }^{1}$, Kristinn B. Gylfason ${ }^{2}$ and Dries Van Thourhout ${ }^{1}$ \\ ${ }^{1}$ Photonics Research Group, Gent University-imec, 9000 Gent, Belgium \\ ${ }^{2}$ KTH - Royal Institute of Technology, SE-100 44 Stockholm, Sweden \\ ${ }^{3}$ Ecole Polytechnique Fédérale de Lausanne (EPFL), 1015 Lausanne, Switzerland \\ ${ }^{4}$ Interuniversity Microelectronics Centre, 3DSIP department, 3001 Leuven, Belgium \\ *Khannan.Rajendran@Ugent.be
}

\begin{abstract}
The thermal motion of a vacuum packaged, suspended nano-beam and nanophotonic waveguide on a silicon photonics-MEMS platform is measured. The resonance frequency and Q-factor of the observed mechanical modes are determined. We also experimentally estimate the displacement sensitivity to be $1.779 \pm 0.022 \frac{\mathrm{fm}}{\sqrt{\mathrm{Hz}}}$.
\end{abstract}

\section{INTRODUCTION}

Silicon photonics is a high-refractive index contrast photonic integrated circuit (PIC) platform that allows large-scale and low cost integration of standardized components. The platform boasts of low loss passives like waveguides, splitters etc. and high performance active components such as modulators, detectors and phase-shifters. Integrating microelectromechanical systems (MEMS) into the existing silicon photonics technology platform, facilitates mechanical motion at the micro- and nanoscale of suspended photonic components with low power operation, low loss and small footprint [1]. Such a platform allows for the fabrication of novel optomechanical devices, to study and exploit the interaction of mechanical and optical waves via radiation pressure. By actuating MEMS, the interaction distance between optical waveguides and nanobeams can be mechanically tuned [1]. This has a strong influence on the opto-mechanical coupling and provides an additional means to control the interaction strength [2].

The thermo-mechanical noise of a vibrating mechanical system is used calibrate its motion. This is performed by invoking the equipartition theorem, which relates the the thermal energy of the device to its mean squared amplitude of motion [3]. Such thermal noise measurements are performed under vacuum conditions, in order to eliminate the air damping of the mechanical motion [2], [4], [5]. The low pressure environment is implemented either by using a vacuum chamber [2], [5] or via vacuum packaging the device [6].

In this paper, we report the design and fabrication of a vacuum encapsulated, suspended silicon nano-beams based optomechanical device. We experimentally observe the different mechanical modes by measuring their thermal noise spectrum. Finally, we determine the minimum measurable displacement of the system.

This work was supported by the EU conmission through Grant Agreement 732894 (FET proactive HOT), 722923 (OMT) and 780283 (MORPHIC)

\section{DEVICE AND FABRICÁTION}

The device is fabricated on imec's iSiPP50G active Silicon photonics platform. It consists of a "W-shaped" bent silicon nanophotonic waveguide $(450 \mathrm{~nm} \times 220 \mathrm{~nm})$ and a thin curved nano-beam $(220 \mathrm{~nm} \times 220 \mathrm{~nm})$ which follows the curvature of the waveguide. The beam is attached to a low stiffness comb-drive, Fig.1b. The waveguide and nano-beam together form an optical phase shifter as shown in schematic Fig.la. To mechanically actuate the MZI arm and the nano-beam, they were suspended by etching the buried oxide $(2 \mu \mathrm{m})$ with vapor HF [1]. Finally, the device is hermetically sealed in a vacuum environment using a thin $\mathrm{Si}$ cap via a wafer-scale thermocompression bonding process [6].

The waveguide supports a single (TE) optical mode, that is evanescently perturbed by the thin nanobeam. The displacement of either the beam or the waveguide relative to each other, results in a phase change. The interferometer is used to convert the phase variations into a transmission change. This is imprinted onto the probe as optical power variations to enable detection by a optical detector.

\section{Mechanics Simulation}

The mechanical vibration modes of the suspended portion of the phase-shifter, that includes one of the MZI arm and a nano-beam close to it, is numerically investigated with the finite element method. A fixed boundary constraint is used at both sides of the waveguide as well as the nano-beam. The corresponding mode profile, frequency $\left(f_{0}^{s i m}\right)$, and the effective mass $\left(m_{e f f}\right)$ is shown in Fig. 2 . The first three modes are in-plane ( $x-y$ plane) vibrations, and the fourth mode shows out-of-plane ( $y-z$ plane) vibration. The nanobeam (NB) modes in Fig. 2 have similar mode profile but slightly different frequencies due to discretization error in the numerical meshing.

\section{MEAsurement SETuP}

The experimental setup consists of a probe $(1543.5 \mathrm{~nm})$ from a Santec TSL510 laser, that is provided into the input grating coupler of the MZI. The output from the MZI is split using a 99:1 splitter. The 1\% tapped optical power is used to monitor the transmission of the device using a power meter. The remaining output power is supplied to a photodetector 

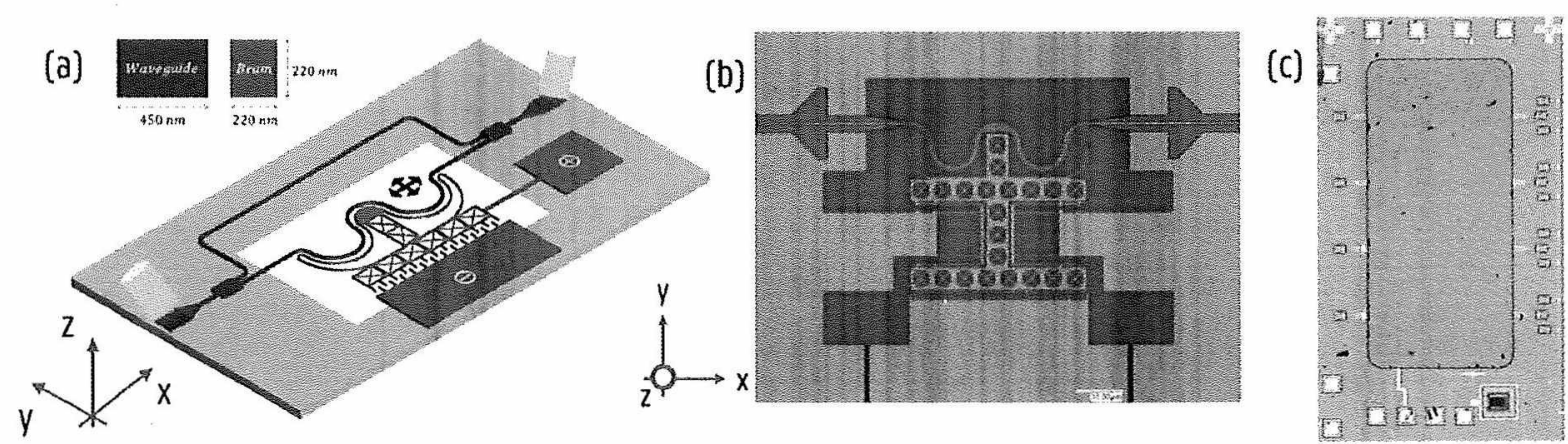

Fig. 1. (a) Schematic of the device. Cross-section profile of the waveguide and nano-beam shown in inset. (b) Microscope image of the suspended arm of the MZI in an unsealed sample. The image shows the waveguide, nano-beam and the comb-drive. Silicon device layer in purple. Under-etched region in dark blue. (c) Microscope image of the MZI, vacuum sealed using a silicon lid.
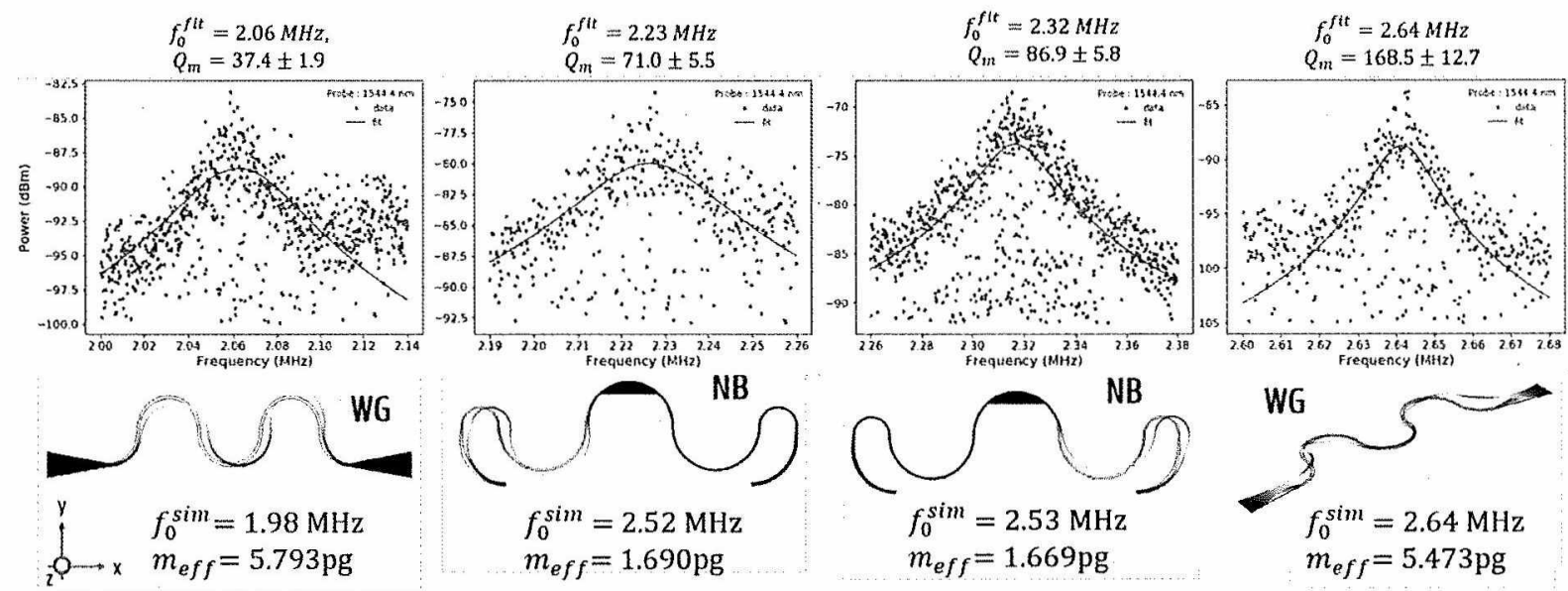

Fig. 2. Measured thermal noise spectrum: The lorentzian fit is shown in red, according to [3]. The fitted resonance frequency $\left(f_{0}^{f i t}\right)$ and nechanical quality factor $\left(Q_{m}\right)$ are indicated at top of the figures. The corresponding simulated mechanical mode profile, resonance frequency $\left(f_{0}^{s i n}\right)$ and effective mass $\left(m_{e} f f\right)$ using COMSOL are shown below. WG: waveguide, NB: nano-beam.

(PD) with a noise equivalent power of $9.3 \frac{\mathrm{pW}}{\sqrt{\mathrm{Hz}}}$ and $1.6 \mathrm{GHz}$ bandwidth. The RF-signal from the PD is recorded using an Agilent N9010A electrical spectrum analyzer (ESA). The resolution and video bandwidth of the ESA are both set to be $24 \mathrm{~Hz}$. The resulting RF spectrum from the vibrating waveguide and nanobeam are shown in Fig. 2. The measurements are performed at $23{ }^{\circ} \mathrm{C}$.

\section{RESULTS}

Using the equipartition theorem, the measured RF power spectral density from the PD can be related to the displacement noise spectral density [3], [4]. The measured RF noise spectrum is fitted using a Lorentzian [3] to determine the mechanical resonance frequencies $\left(f_{0}\right)$ and the mechanical quality factors $\left(Q_{m}\right)$. The measured and simulated mode frequencies show good correspondence, as seen in Fig.2. The $2.64 \mathrm{MHz}$ out-of-plane waveguide mechanical mode exhibits the largest Q-factor of 168.5. The detected white background noise $\left(S_{P}^{W}\right)$ is $-120 \mathrm{dBm}$. From the fitting, we also obtain the transduction factor $(\alpha)$ as $13.16 \pm 0.33 \frac{\mathrm{pW}}{\mathrm{pm}^{2}}$. Where $\alpha$ represents the efficiency of the detection system in converting the displacement of nanobeam/waveguide into the measured RF power. The displacement sensitivity, which gives the minimum detectable displacement of the system, is: $\sqrt{\frac{S_{P}^{W /}}{\alpha}}=1.779 \pm 0.022 \frac{\mathrm{fm}}{\sqrt{\mathrm{Hz}}}$ and is comparable to other MZI [2] and MEMS [5] based opto-mechanical systems.

\section{CONCLUSION}

We have demonstrated an opto-mechanical device comprised of a suspended silicon nano-beam phase shifter on a vacuum packaged, active silicon photonics-MEMS platform. The different mechanical vibrational modes of the nanobeam and waveguide are simulated. The associated resonance frequencies and mechanical quality factors are experimentally measured. We also determine the displacement sensitivity of the system to be $1.779 \pm 0.022 \frac{\mathrm{fm}}{\sqrt{\mathrm{Hz}}}$.

\section{REFERENCES}

[1] Q. Niels, et. al., IEEE J. Quantum Electron., vol. 56, pp. 1-10, 2019.

[2] M. Li, et. al., Nature, vol. 456, pp. 480-484, 2008.

[3] B. D. Hauer, et. al., Annals of Physics, vol. 339, pp. 181-207, 2013

[4] J. Ruels, et. al., Nature Nanotechnolugy, vol. 4, pp. 510-513, 2009.

[5] T. Liu, et. al., Nature communications, vol. 11, pp. 1-7, 2020.

[6] X.Wang et. al., J. Microelectromech. Syst., vol. 28, pp. 460-471, 2019. 


\section{TuC3 - Thermo-mechanical Noise Measurement of Sealed Nanobeams on a Silicon Photonics-MEMS Platform}

ABSTRACT

The thermal motion of a vacuum packaged, suspended nano-beam and nanophotonic waveguide on a silicon photonics-MEMS platform is measured. The resonance frequency and Q-factor of the observed mechanical modes are determined. We also experimentally estimate the displacement sensitivity to be $1.779 \pm 0.022 \mathrm{fm} / \sqrt{ } \mathrm{Hz}$.

TOPICS

Photonic Devices (PD)

MORE IN THIS SESSIOH

Oral : TuC

MC: Oploscousures

10:45am - 11:30am

Tuesday, 7 December 2021

\section{PRESENTED BY}

\section{Khannan Rajendran}

( Belgitum)- Photonics Research Group, IMEC

\section{AUTHORS}

1. Khannan Rajendran (a Belgium) ${ }^{1}$

2. Awanish Pandey (Belgium) ${ }^{1}$

3. Pierre Edinger (m sweden) ${ }^{2}$

4. Gaehun jo (mweden) ${ }^{2}$

5. Alain Yuji Takabayashi (\$ Switzerfand)

6. Umar Khan (" Belgium)

7. Peter Verheyen ( Belgium) ${ }^{4}$

8. Niels Quack ( Switzerland) ${ }^{3}$

9. Frank Niklaus ( Sweden) ${ }^{2}$

10. Wim Bogaerts (a Belgium)

11. Kristinn B. Gylfason ( Sweden) ${ }^{2}$

12. Dries Van Thourhout (Belgium) ${ }^{\prime}$

1. Photonics Research Group, Ghent Univ: $\mathrm{KTH}$ - Royal institute of Technology, 3. Ecc Fédérale de Lausanne (EPF), 4. Interunive Microelectronics Centre, 3DSIP departme: 